\title{
On the Existence of Stable Pairing Distributions
}

\author{
Carlos Castillo-Chavez \\ Biometrics Unit and \\ Center for Applied Mathematics \\ Cornell University \\ Ithaca, NY 14853
}

\author{
Wenzhang Huang \\ Biometrics Unit and \\ Center for Applied Mathematics \\ Cornell University \\ Ithaca, NY 14853
}

\author{
Jia Li \\ Department of Mathematical \\ Sciences \\ Univ. of Alabama at Huntsville \\ Huntsville, AL 35899
}

BU-1242-M

May 1994 


\title{
On The Existence of Stable Pairing Distributions
}

\author{
CARLOS CASTILLO-CHAVEZ* WENZHANG HUANG* JIA LI* $\dagger$
}

\section{Introduction}

According to the American Heritage Dictionary of the English Language (3rd. ed., 1992) demography is the "the study of the characteristics of human populations such as size, growth, density, and vital statistics." Hence it is not surprising that demography has, as an important theoretical component, the field of mathematical demography.

An important aspect of mathematical (which usually includes statistical) demography deals with the development of improved procedures for constructing life tables (invented by the famous astronomer Halley in 1693; as cited in Smith and Keyfitz 1977). As Keyfitz (1977) puts it the "...life table is a scheme for expressing the facts of mortality in terms of probabilities." Milne (1815; as cited in Smith and Keyfitz 1977) adds that "they show the proportion of the people of each sex in every interval of age...". Euler (1760; as cited in Smith and Keyfitz 1977) derived a set of formulas that defined the concept of stable age distribution, that is, a distribution

* Biometrics Unit and Center for Applied Mathematics, Cornell University

$\dagger$ On leave from University of Alabama in Huntsville 
in which the fraction of individuals in each age class remains fixed. He introduced the concept of stable age distribution under the assumptions that the per-capita birth rate is constant while the probabilities of survival remain constant for each age class. Euler used his stable population theory to input missing data. Smith and Keyfitz (1977) stress the importance of this theory by noting that "Euler's virtually unknown article...anticipates important aspects of modern stable population theory for a one-sex population closed to migration."

The connection between stable theory and population dynamics was the result of the efforts of Lotka $(1907,1922)$ and Sharpe and Lotka (1911). The Lotka/Sharpe results were put on a solid mathematical foundation (renewal theory) by Feller (1941). Feller's work continues to generate research activity (see for example, Inaba 1988 and references therein).

One concern of renewal theory is the study of the dynamics of one-sex populations. Our article deals with the study of two-sex populations and their associated stable distributions for populations structured according to fixed characteristics. We illustrate our results with two examples. The structure of these two examples allows for a full analysis a (existence, uniqueness, and stability). The formulation of age-structured models is straightforward (see Castillo-Chavez et al. 1991), but we do not study them because their mathematical analysis is difficult (see Hadeler 1989a, b; Hadeler 1992; Hadeler et al. 1988).

This article is organized as follows: Section 2 briefly reviews the literature on two-sex models and introduces the pair-formation model of Kendall/Keyfitz and Dietz/Hadeler for a homogeneously mixing population as well as the logistic pair-formation model of Castillo-Chavez and Huang (1994). Section 3 partially generalizes the logistic model of Castillo-Chavez and Huang (1994) by incorporating nonrandom mixing in a population structured by fixed characteristics, that is, we move from a single one-group two-sex model to an $n$-group two-sex multigroup heterogeneously mixing model. Section 4 partially analyzes this general $n$-group 
model when all partnerships have the same average duration. Section 5 addresses the problem of uniqueness for stable pair distributions through the discussion of two examples when the total population is bounded. Section 6 introduces a nonlinear $n$ group pair-formation model that generalizes the Kendall/Keyfitz and Dietz/Hadeler one-group model; a multigroup model is formulated in terms of a homogeneous system of order one. Sections 7 and 8 address the problems of existence and stability (trajectorally) of exponential solutions.

\section{Two-Sex Models}

The study of pair-formation or two-sex models in demography was pioneered by Kendall (1949) and Keyfitz (1949). Recent extensions are found in the articles by Fredrickson (1971), McFarland (1972), Parlett (1972), and Pollard (1973). Attempts to understand the basic mechanisms behind the transmission dynamics of the HIV/AIDS epidemic created additional interest in the study of pair-formation models. Increased efforts to understand the dynamics of sexually-transmitted diseases (STD's) have added emphasis to the study of models with partnerships of variable duration (models with multiple simultaneous partnerships are poorly understood, but see Dietz et. al. 1991). Classifications are not usually accurate; however, we roughly identify two theoretical directions in the study of structured populations in demography: the first deals with the study of contact models (a way of studying the effects of high rates of partnership change) while the second deals explicitly with the dynamics of couples (pair-formation models). Both approaches are an attempt to incorporate diverse and complex social dynamics into models for disease spread. Luo and Castillo-Chavez (1991) show that as the rates of pairdissolution approach infinity (that is, as the duration of partnerships approaches zero) pair-formation (and epidemic stochastic) models reduce to contact models (including the general epidemic model of Bailey, see Bailey,1975) with identical pa- 
rameters. Luo and Castillo-Chavez (1991) based their results on the contact/mixing framework developed by Blythe and Castillo-Chavez (1989), Castillo-Chavez and Blythe (1989), Busenberg and Castillo-Chavez (1989, 1991), and Castillo-Chavez and Busenberg (1991). Which model to use and when to use it depends on the type of questions asked and the available data. However, both can be studied under the same theoretical framework.

Extensive connections between social dynamics, demography, social networks, and epidemiology have been encountered over the last few years (see Anderson et al. 1989; Blythe and Castillo-Chavez 1989; Blythe et al. 1991, 1992; Busenberg and Castillo-Chavez 1989, 1991; Castillo-Chavez 1989; Castillo-Chavez et al. 1991; Castillo-Chavez et al. 1994; Dietz 1988; Dietz and Hadeler 1988; Gupta et al. 1989; Hadeler 1989a, b; Hadeler and Nagoma 1990; Huang et al. 1992; Hyman and Stanley 1989; Jacquez et al. 1988, 1989; May and Anderson 1989; Sattenspiel 1987a, b; Sattenspiel and Simon 1988; Sattenspiel and Castillo-Chavez 1990; Waldstätter 1989). Most of these studies (but not all) involve only one-sex populations. In this article, we are concerned exclusively with two-sex models for multigroup populations. We discuss models that support exponential solutions (homogeneous systems of order one) as well as models that only support bounded solutions.

First we introduce the Kendall/Keyfitz and Dietz/Hadeler model (homogeneous system of order one) and include a summary of its properties. Let $m_{s}(t)$ denote the population of single males at time $t, f_{s}(t)$ the population of single females at time $t$, and $p(t)$ the population of paired individuals (heterosexual pairings only) at time $t$. Furthermore, let $\mu_{m}$ and $\mu_{f}$ denote the per-capita removal rates due to death or other factors for males and females; let $\beta_{m}, \beta_{f}$ denote the per-capita birth rates for males and females respectively; and let $\sigma$ denote the per-pair separation rate. With these definitions, one easily derives the following demographic pair-formation model: 


$$
\begin{aligned}
\dot{m}_{s} & =-\mu_{m} m_{s}+\left(\beta_{m}+\mu_{f}+\sigma\right) p-\phi\left(m_{s}, f_{s}\right) \\
\dot{f}_{s} & =-\mu_{f} f_{s}+\left(\beta_{f}+\mu_{m}+\sigma\right) p-\phi\left(m_{s}, f_{s}\right) \\
\dot{p} & =-\left(\mu_{m}+\mu_{f}+\sigma\right) p+\phi\left(m_{s}, f_{s}\right),
\end{aligned}
$$

where $\phi$ denotes the pair-formation rate.

It is assumed that $\phi$ is differentiable for $\left(m_{s}, f_{s}\right) \in \mathbb{R}_{+}^{2} \backslash\{(0,0)\}$ and that it satisfies the properties:

$$
\begin{gathered}
\phi(m, f) \geq 0, \\
\phi(m+u, f+v) \geq \phi(m, f), \\
\phi(\alpha m, \alpha f)=\alpha \phi(m, f), \\
\phi(m, 0)=\phi(0, f)=0 .
\end{gathered}
$$

(See Fredrickson 1971, Hoppensteadt 1975, Dietz 1988, Dietz and Hadeler 1988, Castillo-Chavez et. al. 1994, and references therein.)

System (2.1) and (PF) give a homogeneous system of degree one that supports exponential solutions. Hadeler and collaborators (Hadeler 1989a, b; Hadeler and Nagoma 1990; Hadeler et al. 1988; Hadeler 1992) have developed an extensive theory for homogeneous systems of this type. Using this theory, the following results for system (2.1) have been verified (see Dietz and Hadeler 1988, Waldstätter 1989, and references therein):

I. System (2.1) always has two exponential solutions

$$
m_{s}(t)=e^{-\mu_{m} t}, \quad f_{s}(t)=p(t) \equiv 0,
$$

and

$$
f_{s}(t)=e^{-\mu_{f} t}, \quad m_{s}(t)=p(t) \equiv 0 .
$$

II. If $\phi_{m}$ and $\phi_{f}$ denote the partial derivatives of $\phi$ with respect to the first and the second variable of $\phi$ respectively and if

$$
\mu_{m}<\mu_{f}-\frac{\beta_{f} \phi_{f}(1,0)}{\mu_{f}+\sigma+\phi_{f}(1,0)},
$$


then there is no strictly positive exponential solution, and the exponential solution in $I$. (a) is trajectorally stable (see Hadeler's references for this definition of stability), while the solution expressed in $I .(b)$ is trajectorally unstable.

III. If

$$
\mu_{f}<\mu_{m}-\frac{\beta_{m} \phi_{m}(0,1)}{\mu_{m}+\sigma+\phi_{m}(0,1)},
$$

then there is no strictly positive exponential solution. The exponential solution $I .(b)$ is stable while the exponential solution in $I .(a)$ is unstable.

IV. If

$$
\mu_{m}>\mu_{f}-\frac{\beta_{f} \phi_{f}(1,0)}{\mu_{f}+\sigma+\phi_{f}(1,0)}
$$

and

$$
\mu_{f}>\mu_{m}-\frac{\beta_{m} \phi_{m}(0,1)}{\mu_{m}+\sigma+\phi_{m}(0,1)}
$$

then (2.1) has a unique strictly positive exponential solution, which is stable, while the solutions in $I .(a)$ and $I .(b)$ are both unstable.

Models that also incorporate nonlinear birth and separation processes for twosex homogeneously mixing populations are also being studied. To contrast the effects of mathematical homogeneity of order one, we revisit the extension of the system (2.1) introduced by Castillo-Chavez and Huang (1994). This extension incorporates nonlinear birth and dissolution rates; in fact, it assumes that the birth rate $\beta=\beta(T)$ and the separation rate $\sigma=\sigma(T)$ are functions of the total population $T=m_{s}+f_{s}+2 p$. They also introduce the constants $\gamma$ and $1-\gamma(0<\gamma<1)$, representing the proportions of the male and female birth rates respectively. They arrive at the system:

$$
\begin{aligned}
\dot{m}_{s} & =-\mu_{m} m_{s}+\left[\gamma \beta(T)+\mu_{f}+\sigma(T)\right] p-\phi\left(m_{s}, f_{s}\right) \\
\dot{f}_{s} & =-\mu_{f} f_{s}+\left[(1-\gamma) \beta(T)+\mu_{m}+\sigma(T)\right] p-\phi\left(m_{s}, f_{s}\right) \\
\dot{p} & =-\left[\mu_{m}+\mu_{f}+\sigma(T)\right] p+\phi\left(m_{s}, f_{s}\right),
\end{aligned}
$$

where $\beta$ and $\sigma$ are assumed to satisfy the following properties:

$$
\frac{d \beta(T)}{d T}<0, \quad \lim _{T \rightarrow \infty} \beta(T)=0
$$




$$
\frac{d \sigma(T)}{d T} \geq 0
$$

Castillo-Chavez and Huang (1994) have established the following results:

Result A If either

$$
\min \left\{\frac{\gamma \beta(0)}{\mu_{m}}, \frac{(1-\gamma) \beta(0)}{\mu_{f}}\right\} \leq 1
$$

or

$$
\min \left\{\frac{\gamma \beta(0)}{\mu_{m}}, \frac{(1-\gamma) \beta(0)}{\mu_{f}}\right\}>1
$$

and

$$
\mu_{m}+\mu_{f}+\sigma(0) \geq \phi\left(\frac{\gamma \beta(0)}{\mu_{m}}-1, \frac{(1-\gamma) \beta(0)}{\mu_{m}}-1\right),
$$

then for any given initial condition $z=\left(m_{0}, f_{0}, p_{0}\right) \in \Omega$

$$
\lim _{t \rightarrow \infty} m(t, z)=\lim _{t \rightarrow \infty} f(t, z)=\lim _{t \rightarrow \infty} p(t, z)=0
$$

Condition (2.3) implies that either the male growth rate or the female growth rate is negative, that is, either the total male population or the total female population vanishes and, consequently, so does the total paired population. From Condition $(2.4)_{a}$ it is not obvious that the population goes to zero because both maximum male and female reproductive numbers, $\frac{\gamma \beta(0)}{\mu_{m}}$ and $\frac{(1-\gamma) \beta(0)}{\mu_{f}}$, exceed one. However, Condition $(2.4)_{b}$ roughly implies that the growth rate for paired-individuals is negative and, therefore, the reproduction rates of male and female populations are sufficiently reduced so that extinction takes place.

Result B For system (2.2), if

$$
\begin{gathered}
\min \left\{\frac{\gamma \beta(0)}{\mu_{m}}, \frac{(1-\gamma) \beta(0)}{\mu_{f}}\right\}>1 \\
\mu_{m}+\mu_{f}+\sigma(0)<\phi\left(\frac{\gamma \beta(0)}{\mu_{m}}-1, \frac{(1-\gamma) \beta(0)}{\mu_{m}}-1\right) .
\end{gathered}
$$


then the zero solution is unstable and there exists a unique positive equilibrium $\left(m^{*}, f^{*}, p^{*}\right)$. Furthermore, the unique positive equilibrium is locally asymptotically stable.

If the total population $T$ is small, then Condition (3.5) roughly implies that the average growth rates of male, female, and paired populations are positive. Under these circumstances, the total population persists and we expect the existence of at least one positive steady state. Generalizations of models (2.2) and (2.1) to heterogeneously mixing populations are the subject of further analysis in Sections 3 and 6 respectively.

\section{Multiple Group Pair-Formation Model}

The first general mathematical study of single group, two-sex pair-formation models was carried out by Hadeler and Nagoma (1990) and Hadeler et al. (1988). This analysis was extended to handle models with time delay effects (for example of models derived from pair formation models with age-structure, see Hadeler 1992). However, there was no progress for multiple-group pair-formation models until the recent work of Lubkin and Castillo-Chavez (1993) and Lubkin et al. (1993).

The deterministic two-sex population pair-formation model with $n$ subgroups that we formulate in this section generalizes some of the models of Lubkin and Castillo-Chavez (1993). Our modeling approach combines a property similar to (PF) with the two-sex mixing framework of Castillo-Chavez and Busenberg (1991). We assume that the population under consideration can be stratified into $n$ groups. These groups are defined through the use of assumed fixed characteristics such as race, gender, social status, etc. The following definitions are required for the formulation of this model. Let

$m_{i}=$ number of single males in group $i$, 
$f_{i}=$ number of single females in group $i$,

$P_{i j}=$ number of pairs with type $i$ male and type $j$ female,

$\mu_{m}=$ per-capita male mortality rate,

$\mu_{f}=$ per-capita female mortality rate,

$\Lambda_{i}^{m}=$ recruitment rate of single males of type $i$,

$\Lambda_{i}^{f}=$ recruitment rate of single females of type $i$,

$\sigma_{i j}=$ per-ij-pair separation rate,

$C_{i}=$ per capita pair formation rate of type $i$ males,

$B_{i}=$ per capita pair formation rate of type $i$ females,

$p_{i j}=$ proportion of type- $i$ male pairings which are with $j$ females, given that they paired,

$q_{i j}=$ proportion of type- $i$ female pairings which are with $j$ males, given that they paired.

The deterministic dynamics of a two-sex $n$-group pair-formation model can be described with the above definitions by the following system of differential equations:

$$
\begin{gathered}
\dot{m}_{i}=\Lambda_{i}^{m}-\left(\mu_{m}+C_{i}(m, f)\right) m_{i}+\sum_{j}\left(\mu_{f}+\sigma_{i j}\right) P_{i j} \\
\dot{f}_{i}=\Lambda_{i}^{f}-\left(\mu_{f}+B_{i}(m, f)\right) f_{i}+\sum_{j}\left(\mu_{m}+\sigma_{j i}\right) P_{j i} \\
\dot{P}_{i j}=C_{i}(m, f) m_{i} p_{i j}-\left(\mu_{m}+\mu_{f}+\sigma_{i j}\right) P_{i j}
\end{gathered}
$$

where

$$
m=\left(m_{1}, \cdots, m_{n}\right)^{T}, \quad f=\left(f_{1}, \cdots, f_{n}\right)^{T} .
$$

Since $\sum_{i}^{n} C_{i}(m, f) m_{i}$ and $\sum_{i}^{n} B_{i}(m, f) f_{i}$ represent the total pair-formation rates of males and females respectively, we must have

$$
\sum_{i}^{n} C_{i}(m, f) m_{i}=\sum_{i}^{n} B_{i}(m, f) f_{i}
$$

¿From the definitions of $p_{i j}$ and $q_{i j}$ we must also have

$$
\sum_{j}^{n} p_{i j}=\sum_{j}^{n} q_{i j}=1
$$


and

$$
C_{i}(m, f) m_{i} p_{i j}=B_{j}(m, f) f_{j} q_{j i}
$$

Conditions $(i),(i i)$ and ( $i i i)$ are known as the Mixing Axioms or Mixing Properties (see Castillo-Chavez and Busenberg 1991, Castillo-Chavez et al. 1994, Hsu Schmitz et al. 1993, 1994, and Hsu 1994). Solutions to these axioms have been studied by Castillo-Chavez and Busenberg (1991). They are able to characterize all mixing matrices $p_{i j}^{\prime} s$ and $q_{i j}^{\prime} s$ in terms of $B_{i}, C_{i}, m_{i}$, and $f_{i}$. They also show that the only separable solution $p_{i j}^{\prime} s$ and $q_{i j}^{\prime} s$ to the above properties corresponds to heterogeneous random or proportionate mixing (the so-called Ross Solution; see Castillo-Chavez and Busenberg 1991). They characterization of all solutions is given in terms of multiplicative perturbations of Ross solutions (they are named after Ross because its implicit relationship to the mixing axioms, see Ross 1911). Refinements of these results can be found in Hsu Schmitz et al. (1994), and Hsu Schmitz (1994).

Furthermore, we must still deal with the problem of characterizing the nonlinear pair-formation rates $B_{i}(m, f) f_{i}$ and $C_{i}(m, f) m_{i}$ which in general are complex functions of their arguments. The following required properties for the $B_{i}^{\prime} s$ and the $C_{i}$ seem feasible in general, and in fact, they do not contradict property $(\mathbf{P F})$ in the one group case:

$$
\begin{array}{rlrl}
\frac{\partial\left(C_{i}(m, f) m_{i}\right)}{\partial m_{i}} \geq 0, & \frac{\partial\left(B_{i}(m, f) f_{i}\right)}{\partial f_{i}} \geq 0, & i=1, \cdots, n . \\
\frac{\partial C_{i}(m, f)}{\partial m_{j}} \leq 0, & \frac{\partial C_{i}(m, f)}{\partial f_{i}} \geq 0, & j=1, \cdots, n, \\
\frac{\partial B_{i}(m, f)}{\partial m_{i}} \geq 0, & \frac{\partial B_{i}(m, f)}{\partial f_{i}} \leq 0, \quad j=1, \cdots, n .
\end{array}
$$

Thus, an increase of the type $i$ female population will increase the total pair formation rate $\left(C_{i}(m, f) m_{i}\right)$ for type $i$ males, and an increase in the population of any 
type of males/females will reduce/increase the per capita pair formation rate for males/females. Because $(\mathbf{H})$ seems reasonable, we assume its validity throughout the rest of this article.

Finally, we observe that to model heterogeneous pair-formation, the group pairformation rates $B_{i}(m, f) f_{i}$ and $C_{i}(m, f) m_{i}$ must be distributed per unit of time among the various groups under reasonable mixing/pairing constraints. Hence, we assume that the Mixing Axioms (i)-(iii) hold for the rest of this article. The modeling approach described in this article provides a framework for modeling various other types of biological interactions. Castillo-Chavez et al. (1994) and Blythe et al. (1994) report on applications of this framework to cultural anthropology, food web dynamics, vector-transmitted diseases, population genetics, and distribution theory.

This paper does not provide a complete analysis of the general pair-formation model (3.1). Section 4 discusses model (3.1), assuming constant recruitment rates and equal separation rates, while Sections 6-8 further relax these assumptions.

\section{Pair-Formation Models with Constant Recruitment and Identical Av- erage Duration}

First we study a relatively simple sub-model of system (3.1). We suppose that the separation rates $\sigma_{i j}$ are equal, that is,

$$
\sigma_{i j}=\sigma . \quad i, j=1,2, \cdots, n
$$

We also assume that the recruitments $\Lambda_{i}^{m}, \Lambda_{i}^{f}, i=1, \cdots, n$ are constant. Using these assumptions and the new variables

$$
P_{i}=\sum_{j} P_{i j}, \quad Q_{i}=\sum_{j} P_{j i}, \quad i=1, \cdots, n
$$


we can rewrite System (3.1) in the equivalent form:

$$
\begin{aligned}
\dot{m}_{i} & =\Lambda_{i}^{m}-\left(\mu_{m}+C_{i}(m, f)\right) m_{i}+\left(\mu_{f}+\sigma\right) P_{i} \\
\dot{f}_{i} & =\Lambda_{i}^{f}-\left(\mu_{f}+B_{i}(m, f)\right) f_{i}+\left(\mu_{m}+\sigma\right) Q_{i} \\
\dot{P}_{i} & =C_{i}(m, f) m_{i}-\left(\mu_{m}+\mu_{f}+\sigma\right) P_{i} \\
\dot{Q}_{i} & =B_{i}(m, f) f_{i}-\left(\mu_{m}+\mu_{f}+\sigma\right) Q_{i}
\end{aligned}
$$

¿From (4.1) we conclude immediately that

$$
\frac{d}{d t}\left(m_{i}+P_{i}\right)=\Lambda_{i}^{m}-\mu_{m}\left(m_{i}+P_{i}\right)
$$

and that

$$
\frac{d}{d t}\left(f_{i}+Q_{i}\right)=\Lambda_{i}^{f}-\mu_{f}\left(f_{i}+Q_{i}\right)
$$

Hence, we have

$$
\lim _{t \rightarrow \infty}\left(m_{i}(t)+P_{i}(t)\right)=\frac{\Lambda_{i}^{m}}{\mu_{m}}
$$

and

$$
\lim _{t \rightarrow \infty}\left(f_{i}(t)+Q_{i}(t)\right)=\frac{\Lambda_{i}^{f}}{\mu_{m}}
$$

(Note: $m_{i}+P_{i}$ is the total population of type $i$ males and $f_{i}+Q_{i}$ is the total population of type $i$ females.)

If we now let

$$
m_{i}+P_{i}=\frac{\Lambda_{i}^{m}}{\mu_{m}}, \quad f_{i}+Q_{i}=\frac{\Lambda_{i}^{f}}{\mu_{f}},
$$

then Thieme's results on asymptotically autonomous systems (see Thieme 1992, 1993a,b; Castillo-Chavez and Thieme 1993) make it possible to reduce the study of the dynamics of system (4.1) to the study of the dynamics of the following limiting system:

$$
\begin{gathered}
\dot{m}_{i}=N_{i}^{m}-\left(\mu+C_{i}(m, f)\right) m_{i}:=G_{i}^{m}(m, f) \\
\dot{f}_{i}=N_{i}^{f}-\left(\mu+B_{i}(m, f)\right) f_{i}:=G_{i}^{f}(m, f)
\end{gathered}
$$


where $\mu=\mu_{m}+\mu_{f}+\sigma$ and

$$
N_{i}^{m}=\left(\frac{\mu_{f}+\sigma}{\mu_{m}}+1\right) \Lambda_{i}^{m}, \quad N_{i}^{f}=\left(\frac{\mu_{m}+\sigma}{\mu_{f}}+1\right) \Lambda_{i}^{f} .
$$

Let

$$
\Omega=\left\{(m, f) \in \mathbb{R}_{+}^{2 n} ; \quad 0 \leq m_{i} \leq \frac{N_{i}^{m}}{\mu}, 0 \leq f_{i} \leq \frac{N_{i}^{f}}{\mu}, \quad i=1, \cdots, n\right\}
$$

then $\Omega$ is positively invariant to the flow of (4.3) and any solution with initial value on the boundary of $\Omega$ will eventually enter inside of $\Omega$. Hence, there is at least one equilibrium in the interior of $\Omega$. This is the content of the following theorem:

Theorem 4.1 If (4.3) has only one equilibrium in $\Omega$, then it is a global attractor of $\Omega$.

Proof. Let $G^{m}=\left(G_{1}^{m}, \cdots, G_{n}^{m}\right)^{T}, G^{f}=\left(G_{1}^{f}, \cdots, G_{n}^{f}\right)^{T}$. Then by using the hypotheses $(\mathrm{H})$, we can see that the Jacobian matrix of $\left(G^{m}, G^{f}\right)^{T}$ at $(m, f)$ is of the form

$$
\frac{\partial\left(G^{m}, G^{f}\right)}{\partial(m, f)}=\left[\begin{array}{ll}
W_{11} & W_{12} \\
W_{21} & W_{22}
\end{array}\right]
$$

where $W_{i j} \in \mathbb{R}^{n \times n},(i, j=1,2)$ the off-diagonal entries of $W_{11}, W_{22}$ are nonnegative, and $W_{21}, W_{12}$ are nonpositive matrices for $(m, f) \in \Omega$. Hence, System (4.3) generates a monotone flow. From the results on monotone flows of Smith and Thieme (1990) Theorem (4.1) follows.

Remark: Under hypotheses $(\mathrm{H})$ it has not been possible to prove the existence of a unique positive equilibrium. In fact, we believe that multiple equilibria are possible. Nevertheless, Section 5 provides two examples where uniqueness can be established.

\section{Uniqueness of Stable Paired Distributions: Two Examples}


For the two examples of this section we are able to show the existence of a unique positive stable pairing distribution. These examples consider arbitrary mixing matrices but specific per-capita pairing rates. Example 1 This example uses as functional forms for the per-capita pair-formation rates $C_{i}$ and $B_{i}, i=$ $1, \cdots, n$ the so-called harmonic means

$$
\begin{aligned}
& C_{i}(m, f)=\frac{\alpha_{i} \sum_{k} \beta_{k} f_{k}}{\sum_{k} \alpha_{k} m_{k}+\sum_{k} \beta_{k} f_{k}}, \\
& B_{i}(m, f)=\frac{\beta_{i} \sum_{k} \alpha_{k} m_{k}}{\sum_{k} \alpha_{k} m_{k}+\sum_{k} \beta_{k} f_{k}},
\end{aligned}
$$

or

$$
\begin{aligned}
& C_{i}(m, f)=\alpha_{i} \sum_{k} \frac{\beta_{k} f_{k}}{\alpha_{i} m_{i}+\beta_{k} f_{k}}, \\
& B_{i}(m, f)=\beta_{i} \sum_{k} \frac{\alpha_{k} m_{k}}{\alpha_{k} m_{k}+\beta_{i} f_{i}}
\end{aligned}
$$

where $\alpha_{i}, \beta_{i}, i=1, \cdots, n$ are given positive constants. It is clearly that $C_{i}(m, f)$ and $B_{i}(m, f)$ satisfy the hypotheses $(\mathrm{H})$.

Theorem 5.1 Let $C_{i}(m, f)$ and $B_{i}(m, f)$ be defined as in (5.1), then System (4.3) has a unique equilibrium in $\Omega$.

Proof. $\quad m^{*}=\left(m_{1}^{*}, \cdots, m_{n}^{*}\right)^{T}, f^{*}=\left(f_{1}^{*}, \cdots, f_{n}^{*}\right)^{T}$ is an equilibrium of (4.3) if and only if

$$
\begin{aligned}
& N_{i}^{m}=\left(\mu+\frac{\alpha_{i} \sum_{k} \beta_{k} f_{k}^{*}}{\sum_{k} \alpha_{k} m_{k}^{*}+\sum_{k} \beta_{k} f_{k}^{*}}\right) m_{i}^{*}, \\
& N_{i}^{f}=\left(\mu+\frac{\beta_{i} \sum_{k} \alpha_{k} f_{k}^{*}}{\sum_{k} \alpha_{k} m_{k}^{*}+\sum_{k} \beta_{k} f_{k}^{*}}\right) f_{i}^{*} .
\end{aligned}
$$

If we let

$$
T_{1}=\sum_{k} \alpha_{k} m_{k}^{*}, \quad T_{2}=\sum_{k} \beta_{k} f_{k}^{*}
$$

and substitute (5.3) into (5.2), then we have

$$
N_{i}^{m}=\left(\mu+\frac{\alpha_{i} T_{2}}{T_{1}+T_{2}}\right) m_{i}^{*}, \quad N_{i}^{f}=\left(\mu+\frac{\beta_{i} T_{1}}{T_{1}+T_{2}}\right) f_{i}^{*}, \quad i=1, \cdots, n
$$


¿From (5.4) it follows that

$$
m_{i}^{*}=\frac{N_{i}^{m}\left(T_{1}+T_{2}\right)}{\mu T_{1}+\left(\mu+\alpha_{i}\right) T_{2}}, \quad f_{i}^{*}=\frac{N_{i}^{f}\left(T_{1}+T_{2}\right)}{\mu T_{2}+\left(\mu+\beta_{i}\right) T_{1}} .
$$

Equations (5.3) and (5.5) yield that

$$
\begin{aligned}
& \sum_{k} \frac{\alpha_{k} N_{k}^{m}\left(T_{1}+T_{2}\right)}{\mu T_{1}+\left(\mu+\alpha_{k}\right) T_{2}}-T_{1}=0 \\
& \sum_{k} \frac{\beta_{k} N_{k}^{f}\left(T_{1}+T_{2}\right)}{\mu T_{2}+\left(\mu+\beta_{k}\right) T_{1}}-T_{2}=0 .
\end{aligned}
$$

Conversely, if $T_{1}>0, T_{2}>0$ satisfies Equation (5.6), then setting $m_{i}^{*}, f_{i}^{*}$ as defined in (5.5) gives a solution of (5.2). Therefore, it is enough to prove that (5.6) has a unique solution.

To study the question of the uniqueness of equilibrium to (5.6), we consider the system of differential equations

$$
\begin{aligned}
& \dot{T}_{1}=\sum_{k} \frac{\alpha_{k} N_{k}^{m}\left(T_{1}+T_{2}\right)}{\mu T_{1}+\left(\mu+\alpha_{k}\right) T_{2}}-T_{1} \\
& \dot{T}_{2}=\sum_{k} \frac{\beta_{k} N_{k}^{f}\left(T_{1}+T_{2}\right)}{\mu T_{2}+\left(\mu+\beta_{k}\right) T_{1}}-T_{2} .
\end{aligned}
$$

System (5.7) has a positively invariant set,

$$
\Sigma=\left\{\left(T_{1}, T_{2}\right) \in \mathbb{R}_{+}^{2} ; 0 \leq T_{1} \leq \sum_{k} \frac{\alpha_{k} N_{k}^{m}}{\mu}, 0 \leq T_{2} \leq \sum_{k} \frac{\beta_{k} N_{k}^{f}}{\mu}\right\}
$$

which is an attractor of all points in $\mathbb{R}_{+}^{2}$. Furthermore, a solution of (5.6) in $\mathbb{R}_{+}^{2}$ must be an equilibrium of (5.7) in $\Sigma$.

We claim that any equilibrium of (5.7) in $\Sigma$ is asymptotically stable. In fact, if $T_{1}^{*}, T_{2}^{*}$ is an equilibrium, then the Jacobian matrix at $T_{1}^{*}, T_{2}^{*}$ is

$$
J=\left[\begin{array}{cc}
-1+\sum_{k} \frac{\alpha_{k}^{2} N_{k}^{m} T_{2}^{*}}{\left[\mu T_{1}^{*}+\left(\mu+\alpha_{k}\right) T_{2}^{*}\right]^{2}} & -\sum_{k} \frac{\alpha_{k}^{2} N_{k}^{m} T_{1}^{*}}{\left[\mu T_{1}^{*}+\left(\mu+\alpha_{k}\right) T_{2}^{*}\right]^{2}} \\
-\sum_{k} \frac{\beta_{k}^{2} N_{i}^{f} T_{2}^{*}}{\left[\mu T_{2}^{*}+\left(\mu+\beta_{k}\right) T_{1}^{*}\right]^{2}} & -1+\sum_{k} \frac{\beta_{k}^{2} N_{i}^{f} T_{1}^{*}}{\left[\mu T_{2}^{*}+\left(\mu+\beta_{k}\right) T_{1}^{*}\right]^{2}}
\end{array}\right]
$$


Since

$$
\begin{aligned}
& \operatorname{tr}(J)=-2+\sum_{k} \frac{\alpha_{k}^{2} N_{k}^{m} T_{2}^{*}}{\left[\mu T_{1}^{*}+\left(\mu+\alpha_{k}\right) T_{2}^{*}\right]^{2}}+\sum_{k} \frac{\beta_{k}^{2} N_{k}^{f} T_{1}^{*}}{\left[\mu T_{2}^{*}+\left(\mu+\beta_{k}\right) T_{1}^{*}\right]^{2}} \\
&=-2+\sum_{k} \frac{\alpha_{k} N_{k}^{m}}{\left[\mu T_{1}^{*}+\left(\mu+\alpha_{k}\right) T_{2}^{*}\right]} \frac{\alpha_{k} T_{2}^{*}}{\left[\mu T_{1}^{*}+\left(\mu+\alpha_{k}\right) T_{2}^{*}\right]} \\
&+\sum_{k} \frac{\beta_{k} N_{k}^{f}}{\left[\mu T_{2}^{*}+\left(\mu+\beta_{k}\right) T_{1}^{*}\right]} \frac{\beta_{k} T_{1}^{*}}{\left[\mu T_{2}^{*}+\left(\mu+\beta_{k}\right) T_{1}^{*}\right]} \\
& \leq-2+\sum_{k} \frac{\alpha_{k} N_{k}^{m}}{\left[\mu T_{1}^{*}+\left(\mu+\alpha_{k}\right) T_{2}^{*}\right]}+\sum_{k} \frac{\beta_{k} N_{i}^{f}}{\left[\mu T_{2}^{*}+\left(\mu+\beta_{k}\right) T_{1}^{*}\right]} \\
&=-2+\frac{T_{1}^{*}}{T_{1}^{*}+T_{2}^{*}}+\frac{T_{1}^{*}}{T_{1}^{*}+T_{2}^{*}} \\
&=-1 \\
&<0
\end{aligned}
$$

and

$$
\begin{aligned}
\operatorname{det}(J)= & \left.-1+\sum_{k} \frac{\alpha_{k}^{2} N_{k}^{m} T_{2}^{*}}{\left[\mu T_{1}^{*}+\left(\mu+\alpha_{k}\right) T_{2}^{*}\right]^{2}}\right)\left(-1+\sum_{k} \frac{\beta_{k}^{2} N_{k}^{f} T_{1}^{*}}{\left[\mu T_{2}^{*}+\left(\mu+\beta_{k}\right) T_{1}^{*}\right]^{2}}\right) \\
& -\left(\sum_{k} \frac{\alpha_{k}^{2} N_{k}^{m} T_{2}^{*}}{\left[\mu T_{1}^{*}+\left(\mu+\alpha_{k}\right) T_{2}^{*}\right]^{2}}\right)\left(\sum_{k} \frac{\beta_{k}^{2} N_{k}^{f} T_{1}^{*}}{\left[\mu T_{2}^{*}+\left(\mu+\beta_{k}\right) T_{1}^{*}\right]^{2}}\right) \\
= & 1-\sum_{k} \frac{\alpha_{k}^{2} N_{k}^{m} T_{2}^{*}}{\left[\mu T_{1}^{*}+\left(\mu+\alpha_{k}\right) T_{2}^{*}\right]^{2}}-\sum_{k} \frac{\beta_{k}^{2} N_{k}^{f} T_{1}^{*}}{\left[\mu T_{2}^{*}+\left(\mu+\beta_{k}\right) T_{1}^{*}\right]^{2}} \\
> & 1-\sum_{k} \frac{\alpha_{k} N_{k}^{m}}{\left[\mu T_{1}^{*}+\left(\mu+\alpha_{k}\right) T_{2}^{*}\right]}-\sum_{k} \frac{\beta_{k} N_{k}^{f}}{\left[\mu T_{2}^{*}+\left(\mu+\beta_{k}\right) T_{1}^{*}\right]} \\
= & 0,
\end{aligned}
$$

we conclude, from elementary ODE theory, that the equilibrium $\left(T_{1}^{*}, T_{2}^{*}\right)$ is locally asymptotically stable. Furthermore, since (5.7) is a competitive system, there are no periodic solutions in $\Sigma$ (Hirsch 1982). It follows from two-dimensional ODE theory (planar systems) that there is only one equilibrium in $\Sigma$. Hence, System (5.6) has a unique solution. This completes the proof.

Putting Theorem 4.1 and 5.1 together we conclude: 
Theorem 5.2 Suppose $\sigma_{i j}=\sigma, i, j=1, \cdots, n$ and $C_{i}(m, f), B_{i}(m, f)$ are given by (5.1), then the system (3.1) has a unique positive equilibrium which is a global attractor of all nonnegative solutions.

Remark 1 Theorem 5.2 also holds if $C_{i}(m, f)$ and $B_{i}(m, f)$ are given by $(5.1)^{*}$. However, we omit the proof.

Example 2 Consider the case where females choose their partners, that is, males get chosen at a constant per-female pairing rate. We assume that the per-capita rate of pair formation of type $i$ females is

$$
B_{i}(m, f)=b_{i}=\text { constant }, \quad i=1, \cdots, n .
$$

Hence, from Mixing Axiom (iii), it follows that $C_{i}(m, f)$ cannot be, in general, constant. A valid selection must incorporate, into the definition of the per-capita rate of pair-formation of type $i$ males, the effects a variable partnership pool. A choice of a multiplier, that is, a selection that guarantees that the Mixing Axioms are satisfied, is given by the sexual-activity ratio. Explicitly, we let

$$
C_{i}(m, f)=c_{i} \frac{\sum_{k=1}^{n} b_{k} f_{k}}{\sum_{k=1}^{n} c_{k} m_{k}}, \quad i=1 \cdots, n
$$

where $c_{1}, \cdots, c_{n}$ are positive constants. These per-capita pair-formation rates $B_{i}$ and $C_{i}$ do not contradict the Mixing Axioms and hypothesis $(\mathbf{H})$. However, we must be aware that $C_{i}(m, f)$ will be unbounded as $m \rightarrow 0$, that is, solutions could go to zero or even become negative. In order to avoid this situation, we add constraints to the selection of the constants $b_{i}$ and $c_{i}$. Explicitly, we assume that the dominant eigenvalue of the matrix

$$
M=\operatorname{diag}\left(N_{i}^{m}\right)\left[\begin{array}{ccc}
c_{1} & \cdots & c_{n} \\
\cdot & \cdots & \cdot \\
c_{1} & \cdots & c_{n}
\end{array}\right]-\operatorname{diag}\left(c_{i} \sum_{k=1}^{n} b_{k} \frac{N_{k}^{f}}{\mu+b_{k}}\right)
$$


is positive. Next, we show that Equation (4.3) has a unique positive equilibrium when $B_{i}$ 's and $C_{i}$ 's are defined by $(5.8)-(5.9)$ and $M$ has a positive dominant eigenvalue.

Since $B_{i}=b_{i}$ is a constant for each $i$ then the equilibrium

$$
\left(m^{*}, f^{*}\right)=\left(m_{i}^{*}, \cdots, m_{n}^{*}, f_{i}^{*}, \cdots, f_{n}^{*}\right)
$$

must satisfy

$$
f_{i}^{*}=\frac{N_{i}^{f}}{\mu+b_{i}} .
$$

Moreover, $m^{*}$ satisfies the system

$$
N_{i}^{m}-\left(\mu+c_{i} \frac{\sum_{k=1}^{n} b_{k} f_{k}^{*}}{\sum_{k=1}^{n} c_{k} m_{k}}\right) m_{i}=0, \quad i=1, \cdots, n,
$$

or, more succinctly, the system

$$
S(m)=0,
$$

where the $i$ th component of $S$ takes the form

$$
S_{i}(m)=\left(N_{i}^{m}-\mu m_{i}\right) \sum_{k=1}^{n} c_{k} m_{k}-c_{i}\left(\sum_{k=1}^{n} b_{k} f_{k}^{*}\right) m_{i} . \quad i=1, \cdots, n .
$$

The function $S$ (from a well known result) has a strictly positive zero if and only if the dominant eigenvalue of

$$
D S(0)=\left(\frac{\partial S_{i}(0)}{\partial m_{j}}\right)_{n \times n}=M
$$

is positive. Consequently, we have established the following result:

Theorem 5.3 Let $B_{i}(m, f)$ and $C_{i}(m, f)$ be defined by (5.8) - (5.9) and assume that the matrix in (5.10) has a positive dominant eigenvalue, then System (4.1) has a unique positive equilibrium which is globally asymptotically stable.

A similar result is obtained if the roles of male and female are reversed. 
Remark 2 When System (4.1) has a unique positive equilibrium we can also obtain a useful relationship between the asymptotic solutions and the asymptotic mixing matrices: A stable pairing distribution. The argument goes as follows: from system (3.1) we notice that $P_{i j}$, the number of pairs of type $i$ male and type $j$ females, satisfies the equation

$$
\dot{P}_{i j}=C_{i}(m, f) m_{i} p_{i j}-\left(\mu_{m}+\mu_{f}+\sigma\right) P_{i j} .
$$

Using the formula of variation of constants and letting $t \rightarrow \infty$, we obtain

$$
\begin{aligned}
P_{i j}(t)= & P_{i j}(0) e^{-\left(\mu_{m}+\mu_{f}+\sigma\right) t} \\
& +\int_{0}^{t} e^{-\left(\mu_{m}+\mu_{f}+\sigma\right)(t-s)} C_{i}(m(s), f(s)) m_{i}(s) p_{i j}(m(s), f(s)) d s \\
& \rightarrow \frac{C_{i}\left(m^{*}, f^{*}\right) m_{i}^{*} p_{i j}\left(m^{*}, f^{*}\right)}{\mu_{m}+\mu_{f}+\sigma} \\
= & \frac{B_{j}\left(m^{*}, f^{*}\right) f_{j}^{*} q_{j i}\left(m^{*}, f^{*}\right)}{\mu_{m}+\mu_{f}+\sigma},
\end{aligned}
$$

where $\left(m_{1}^{*}, \cdots, m_{n}^{*}\right)$ and $\left(f_{1}^{*}, \cdots, f_{n}^{*}\right)$ denote the values of the unique positive equilibrium. It follows that

$$
\lim _{t \rightarrow \infty} \frac{P_{i j}(t)}{\sum_{j} P_{i j}(t)}=p_{i j}\left(m^{*}, f^{*}\right),
$$

and

$$
\lim _{t \rightarrow \infty} \frac{P_{i j}(t)}{\sum_{j} P_{j i}(t)}=q_{j i}\left(m^{*}, f^{*}\right),
$$

that is, the stable pairing distribution coincides with the mixing matrix. In particular, if $p_{i j}$ and $q_{i j}$ represent random or proportionate mixing (Ross solutions, see Castillo-Chavez and Busenberg 1991), that is, if

$$
\bar{p}_{i}(m, f)=\frac{B_{i}(m, f) f_{i}}{\sum_{k} B_{k}(m, f) f_{k}},
$$

and 


$$
\bar{q}_{j}(m, f)=\frac{C_{j}(m, f) m_{j}}{\sum_{k} C_{k}(m, f) m_{k}}
$$

then

$$
\lim _{t \rightarrow \infty} \frac{P_{i j}(t)}{\sum_{i} \sum_{j} P_{i j}(t)}=\bar{p}_{i}\left(m^{*}, f^{*}\right) \bar{q}_{j}\left(m^{*}, f^{*}\right) .
$$

Then we have stochastic independence.

\section{Pair-formation Models with Constant Per-Pair Birth or Recruitment Rates}

We now turn to the model (4.1) with nonconstant recruitments $\Lambda_{i}^{m}, \Lambda_{i}^{f}$. Suppose that females and males have equal birth rates, $2 k$, and that a couple of a type $i$ male and a type $j$ female have the same probability of producing male and female offspring of a given type. Then under this assumption, $\Lambda_{i}^{m}$ and $\Lambda_{i}^{f}$ are given by

$$
\Lambda_{i}^{m}=\Lambda_{i}^{f}=k\left(P_{i}+Q_{i}\right)
$$

Let us further assume that $\mu_{m}=\mu_{f}=\mu$, then System (4.1) takes the form

$$
\begin{aligned}
& \dot{m}_{i}=-\left(\mu+C_{i}(m, f)\right) m_{i}+(\mu+\sigma+k) P_{i}+k Q_{i} \\
& \dot{f}_{i}=-\left(\mu+B_{i}(m, f)\right) f_{i}+k P_{i}+(\mu+\sigma+k) Q_{i} \\
& \dot{P}_{i}=C_{i}(m, f) m_{i}-(\mu+\mu+\sigma) P_{i} \\
& \dot{Q}_{i}=B_{i}(m, f) f_{i}-(\mu+\mu+\sigma) Q_{i} .
\end{aligned}
$$

If $B_{i}(m, f), C_{i}(m, f)$, the per-capita pair-formation rate of $i$ females and males are homogeneous functions of degree zero, that is, for any nonnegative number $\alpha$,

$$
B_{i}(\alpha m, \alpha f)=B_{i}(m, f) \quad \text { and } \quad C_{i}(\alpha m, \alpha f)=C_{i}(m, f)
$$

Then System (6.1) is a homogeneous system of degree one. Following Kendall (1949), Keyfitz (1949), Fredrickson (1971), McFarland (1972), Pollard (1973), and 
Dietz and Hadeler (1988), we investigate the existence of nonnegative exponential solutions. Complete results (existence, uniqueness, and stability of solutions) have been studied by Dietz and Hadeler (1988) only for the one-group model given by System (2.1). This analysis is based on the monotonicity of System (2.1), a property that does not hold for System (4.1). Hence geometrical or topological methods cannot be readily applied. Algebraic methods become increasingly inefficient as the dimension of (6.1) increases rapidly with group size. Therefore, because there are no standard procedures for the study of System (6.1), we concentrate on the analysis of special cases.

\section{Existence and Stability of Exponential Solutions}

In this section we investigate the existence of special exponential solutions to System (6.1) as well as their stability using the functional forms defined in (5.1) for $B_{i}(m, f)$ and $C_{i}(m, f)$.

If for each $i, 1 \leq i \leq n$, we let

$$
\begin{gathered}
\Delta_{i}=\left\{(m, f, P, Q) \in \mathbb{R}^{4 n}, m_{i} \geq 0, f_{i} \geq 0, P_{i} \geq 0, Q_{i} \geq 0,\right. \\
\left.m_{j}=f_{j}=P_{j}=Q_{j}=0, j \neq i\right\},
\end{gathered}
$$

then $\Delta_{i}$ is a positively invariant set of (6.1). Furthermore, the orbits in $\Delta_{i}$ are given by solutions to the equations

$$
\begin{aligned}
\dot{m}_{i} & =-\left(\mu+\frac{\alpha_{i} \beta_{i} f_{i}}{\alpha_{i} m_{i}+\beta_{i} f_{i}}\right) m_{i}+(\mu+\sigma+2 k) P_{i} \\
\dot{f}_{i} & =-\left(\mu+\frac{\beta_{i} \alpha_{i} m_{i}}{\alpha_{i} m_{i}+\beta_{i} f_{i}}\right) f_{i}+(\mu+\sigma+2 k) P_{i} \\
\dot{P}_{i} & =\frac{\alpha_{i} \beta_{i} f_{i} m_{i}}{\alpha_{i} m_{i}+\beta_{i} f_{i}}-(2 \mu+\sigma) P_{i}, \\
Q_{i} & =P_{i} .
\end{aligned}
$$

Equation (7.1) is a special case of those discussed in $[\mathrm{H}]$. Therefore, there are two 
exponential solutions:

$$
m_{i}(t)=e^{-\mu t}, \quad f_{i}(t)=P_{i}(t)=0,
$$

and

$$
f_{i}(t)=e^{-\mu t}, \quad m_{i}(t)=P_{i}(t)=0 .
$$

These exponential solutions live in the boundary of $\Delta_{i}$ and are trajectorally unstable. Furthermore, (7.1) has a unique exponential solution-up to a multiplicative positive constant-given by

$$
\begin{gathered}
m_{i}(t)=f_{i}(t)=e^{\lambda_{i} t} \\
P_{i}(t)=Q_{i}(t)=\frac{\alpha_{i} \beta_{i}}{(\lambda+2 \mu+\sigma)\left(\alpha_{i}+\beta_{i}\right)} e^{\lambda_{i} t}
\end{gathered}
$$

where

$$
\lambda_{i}=\frac{1}{2}\left[\sqrt{\left[\frac{\alpha_{i} \beta_{i}}{\alpha_{i}+\beta_{i}}+\mu+\sigma\right]^{2}+\frac{4 k \alpha_{i} \beta_{i}}{\alpha_{i}+\beta_{i}}}-\frac{\alpha_{i} \beta_{i}}{\alpha_{i}+\beta_{i}}-3 \mu-\sigma\right] .
$$

This solution lives in the interior of $\Delta_{i}$ where it is globally trajectorally stable.

To study the local stability of $E_{i} e^{\lambda_{i} t}$ with respect to positive solutions of the full System(6.1), that is, solutions where $E_{i}=\left(E_{i}^{1}, \cdots, E_{i}^{n}\right)$, with

$$
E_{i}^{j}=\left(m_{j}^{i *}, f_{j}^{i *}, P_{j}^{i *}, Q_{j}^{i *}\right) \in \mathbb{R}_{+}^{4}, \quad j=1, \cdots, n,
$$

and

$$
\begin{gathered}
m_{i}^{i *}=f_{i}^{i *}=1, \quad P_{i}^{i *}=Q_{i}^{i *}=\frac{\alpha_{i} \beta_{i}}{(\lambda+2 \mu+\sigma)\left(\alpha_{i}+\beta_{i}\right)} \\
m_{j}^{i *}=f_{j}^{i *}=P_{j}^{i *}=Q_{j}^{i *}=0, \quad j \neq i,
\end{gathered}
$$

we need to compute the Jacobian matrix $J_{i}$ of the functions defined by the right side of (6.1) at $E_{i}$. 
It is not difficult to verify that

$$
J_{i}=\left[\begin{array}{cccc}
J_{1}^{i} & & & \\
& J_{2}^{i} & & \\
& & \ddots & \\
& & & J_{n}^{i}
\end{array}\right],
$$

where

$$
J_{j}^{i}=\left[\begin{array}{cccc}
-\left(\mu+\frac{\alpha_{j} \beta_{i}}{\alpha_{i}+\beta_{i}}\right) & 0 & \mu+\sigma+k & +k \\
0 & -\left(\mu+\frac{\beta_{j} \alpha_{i}}{\alpha_{i}+\beta_{i}}\right) & k & \mu+\sigma+k \\
\frac{\alpha_{j} \beta_{i}}{\alpha_{i}+\beta_{i}} & 0 & -(2 \mu+\sigma) & 0 \\
0 & \frac{\beta_{j} \alpha_{i}}{\alpha_{i}+\beta_{i}} & 0 & -(2 \mu+\sigma)
\end{array}\right] \text {. }
$$

We have $\left[J_{i}^{i}-\lambda_{i} I\right] E_{i}^{i}=0$, and therefore $\left[J_{i}-\lambda_{i} I\right] E_{i}=0$. Furthermore, since $J_{i}^{i}$ is irreducible, the off-diagonal entries of $J_{i}^{i}$ are nonnegative, and $E_{i}^{i}$ is strictly positive then, from a well known result, it follows that $\lambda_{i}$ is a simple eigenvalue of $J_{i}^{i}$, which has the largest real part. The following Lemma due to Waldstätter (1989) provides a criteria for the stability of $E_{i} e^{\lambda_{i} t}$ :

Lemma 7.1 The exponential solution $E_{i} e^{\lambda_{i} t}$ is locally asymptotically stable if

1. $\lambda_{i}$ is a simple eigenvalue of $J_{i}$.

2. $\operatorname{Re}\left(\lambda_{i}-\lambda\right)>0$ for any other eigenvalue $\lambda$ of $J_{i}$.

The exponential solution $E_{i} e^{\lambda_{i} t}$ is unstable if there is a $\lambda \in \sigma\left(J_{i}\right)$ such that $\operatorname{Re}\left(\lambda_{i}-\right.$ $\lambda)<0$.

Applying this result to System (7.1) we conclude that

Theorem 7.2 The exponential solution $E_{i} e^{\lambda_{i} t}$ is locally asymptotically stable if and only if

$$
\begin{gathered}
R_{j}^{i}=\left(\lambda_{i}+2 \mu+\sigma\right)\left(\alpha_{j} \beta_{i}+\beta_{j} \alpha_{i}-2 \alpha_{i} \beta_{i}\right)-\frac{\beta_{i} \alpha_{i}}{\alpha_{i}+\beta_{i}}\left(\alpha_{j} \beta_{i}+\beta_{j} \alpha_{i}-2 \alpha_{j} \beta_{j}\right)<0 \\
1 \leq j \leq n . \quad j \neq i .
\end{gathered}
$$

Furthermore, $E_{i} e^{\lambda_{i} t}$ is unstable if there is $j \neq i$, such that

$$
R_{j}^{i}>0 .
$$


Proof. We notice that the eigenvalues of $J_{j}^{i}$ are the eigenvalues of $J_{i}$ and viceversa. The matrices $J_{j}^{i}, j=1, \cdots, n$ are irreducible with off-diagonal nonnegative entries. Then each $J_{j}^{i}$ has a strictly positive eigenvector $\eta=(x, y, p, q) \in \mathbb{R}^{4}$ associated with its corresponding real dominant eigenvalue $\lambda_{j}^{i}$. Expanding $\left[J_{j}^{i}-\lambda_{j}^{i}\right] \eta=0$ gives

$$
\begin{gathered}
-\left(\lambda+\mu+a_{i j}\right) x+(\mu+\sigma+k) p+k q=0 \\
-\left(\lambda+\mu+b_{i j}\right) y+k p+(\mu+\sigma+k) q=0 \\
a_{i j} x-(\lambda+2 \mu+\sigma) p=0 \\
b_{i j} y-(\lambda+2 \mu+\sigma) q=0,
\end{gathered}
$$

where

$$
a_{i j}=\frac{\alpha_{j} \beta_{i}}{\alpha_{i}+\beta_{i}}, \quad b_{i j}=\frac{\alpha_{i} \beta_{j}}{\alpha_{i}+\beta_{i}} .
$$

Let $x+p=u$ and $y+q=v$. Then by adding the first equation to third and the second to the fourth in (7.2) respectively, we obtain the following expressions:

$$
\begin{gathered}
\left(\lambda_{j}^{i}+\mu\right) u=k(p+q), \\
\left(\lambda_{j}^{i}+\mu\right) v=k(p+q), \\
a_{i j} u=\left(\lambda_{j}^{i}+2 \mu+\sigma+\alpha_{i j}\right) p, \\
b_{i j} v=\left(\lambda_{j}^{i}+2 \mu+\sigma+\beta_{i j}\right) q .
\end{gathered}
$$

¿From (7.3) and (7.4) it follows that

$$
\lambda_{j}^{i}+\mu>0 \quad \text { and } \quad u=v .
$$

Moreover, (7.5) and (7.6) yield that

$$
p=\frac{a_{i j} u}{\lambda_{j}^{i}+2 \mu+\sigma+a_{i j}}, \quad \text { and } q=\frac{b_{i j} u}{\lambda_{j}^{i}+2 \mu+\sigma+b_{i j}} .
$$


The substitution of (7.8) into (7.3) leads to

$$
z_{j}^{i}-k\left(\frac{a_{i j}}{z_{j}^{i}+h+a_{i j}}+\frac{b_{i j}}{z_{j}^{i}+h+b_{i j}}\right)=0,
$$

where $z_{j}^{i}=\lambda_{j}^{i}+\mu$ and $h=\sigma+\mu$ and to

$$
z_{i}-\frac{2 k a_{i i}}{z_{i}+h+a_{i i}}=0, \quad \text { where } z_{i}=\lambda_{i}+\mu .
$$

A straightforward computation shows that

$$
\begin{aligned}
\frac{a_{i j}}{z_{i}+h+a_{i j}} & +\frac{b_{i j}}{z_{i}+h+b_{i j}}-\frac{2 k a_{i i}}{z_{i}+h+a_{i i}} \\
& =\frac{z_{i}+h}{\alpha_{i}+\beta_{i}}\left[\left(z_{i}+h\right)\left(\alpha_{j} \beta_{i}-2 \alpha_{i} \beta_{i}\right)-\frac{\alpha_{i} \beta_{i}}{\alpha_{i}+\beta_{i}}\left(\alpha_{j} \beta_{i}-2 \alpha_{j} \beta_{j}\right)\right] \\
& =\frac{z_{i}+h}{\alpha_{i}+\beta_{i}} R_{j}^{i} .
\end{aligned}
$$

Hence, if we let

$$
F(z, a, b)=z-k\left(\frac{a}{z+h+a}+\frac{b}{z+h+b}\right), \quad z>0, \quad a>0, b>0,
$$

then for any fixed $a$ and $b, F(z, a, b)$ is strictly increasing with respect to $z$. If $R_{j}^{i}<0, j \neq i$ then

$$
F\left(z_{i}, a_{i j}, b_{i j}\right)>z_{i}-\frac{2 k a_{i i}}{z_{i}+h+a_{i i}}=0=F\left(z_{j}^{i}, a_{i j}, b_{i j}\right) .
$$

Therefore, the monotonicity of $F$ implies that $z_{j}^{i}<z_{i}$, and hence that

$$
\lambda_{j}^{i}<\lambda_{i}, \quad \text { if } R_{j}^{i}<0
$$

Similarly, we show that

$$
\lambda_{j}^{i}>\lambda_{i}, \quad \text { if } R_{j}^{i}>0,
$$

and Theorem 7.2 follows immediately from Lemma 7.1 and the Inequalities (7.9) (7.10). 
CASTILLO-CHAVEZ, C. HUANG, W. LI, J.

Next explore the conditions under which System (6.1) has strictly positive exponential solutions (that is, with all positive components). We point out here that, as in the one group model (see Dietz and Hadeler 1988), this type of exponential solution (with all positive components) does not necessarily exist. The problem of determining the number of strictly positive exponential solutions of System (7.1) and their trajectorally stability seems quite difficult. As a first step, we illustrate the complexities associated with this problem through the analysis of two-group models.

\section{Existence and Non-Existence of a Strictly Positive Exponential: The case $n=2$.}

If we let $m_{i}(t)=x_{i} e^{\lambda t}, f_{i}(t)=y_{i} e^{\lambda t}, P_{i}(t)=p_{i} e^{\lambda t}, Q_{i}(t)=q_{i} e^{\lambda t}, i=1,2$ then it represents an exponential solution of (6.1) if and only if $\lambda$ and $x_{i}, y_{i}, p_{i}, q_{i}$ satisfy the system

$$
\begin{gathered}
-\left(\lambda+\mu+\frac{\alpha_{i} \sum_{k}^{2} \beta_{k} y_{k}}{\sum_{k}^{2}\left(\alpha_{k} x_{k}+\beta_{k} y_{k}\right)}\right) x_{i}+(\mu+\sigma+k) p_{i}+k q_{i}=0 \\
-\left(\lambda+\mu+\frac{\beta_{i} \sum_{k}^{2} \alpha_{k} x_{k}}{\sum_{k}^{2}\left(\alpha_{k} x_{k}+\beta_{k} y_{k}\right)}\right) y_{i}+k p_{i}+(\mu+\sigma+k) q_{i}=0 \\
\frac{\alpha_{i} x_{i} \sum_{k}^{2} \beta_{k} y_{k}}{\sum_{k}^{2}\left(\alpha_{k} x_{k}+\beta_{k} y_{k}\right)}-(\lambda+2 \mu+\sigma) p_{i}=0 \\
\frac{\beta_{i} y_{i} \sum_{k}^{2} \alpha_{k} x_{k}}{\sum_{k}^{2}\left(\alpha_{k} x_{k}+\beta_{k} y_{k}\right)}-(\lambda+2 \mu+\sigma) q_{i}=0, \quad i=1,2 .
\end{gathered}
$$

The conditions under which System (8.1) has a strictly positive solution are stated in the following lemma:

Lemma 8.1 System (8.1) has a solution $x_{i}>0, y_{i}>0, p_{i}>0, q_{i}>0, i=1,2$ for some real number $\lambda$ if and only if $\lambda+\mu>0$ and if there is a number $T$ between 
$\frac{\beta_{1}}{\beta_{1}+\alpha_{1}}$ and $\frac{\beta_{2}}{\beta_{2}+\alpha_{2}}$ such that for $i=1,2$,

$$
\lambda+\mu-k\left(\frac{\alpha_{i} T}{\lambda+\mu+h+\alpha_{i} T}+\frac{\beta_{i}(1-T)}{\lambda+\mu+h+\beta_{i}(1-T)}\right)=0,
$$

where $h=\mu+\sigma$.

The proof of Lemma 8.1 is given in the Appendix.

An easy consequence of Lemma 8.1 is

Corollary 8.2 If $\alpha_{1}>\alpha_{2}, \beta_{1}>\beta_{2}$ or $\alpha_{2}>\alpha_{1}, \beta_{2}>\beta_{1}$, then there is no positive exponential solution.

Proof. Suppose $\alpha_{1}>\alpha_{2}, \beta_{1}>\beta_{2}$. Then for any $\lambda$ with $\lambda+\mu=z>0$ and $T \in(0,1)$, we have

$$
\frac{\alpha_{1} T}{z+h+\alpha_{i} T}+\frac{\beta_{1}(1-T)}{z+h+\beta_{1}(1-T)}>\frac{\alpha_{i} T}{z+h+\alpha_{2} T}+\frac{\beta_{2}(1-T)}{z+h+\beta_{2}(1-T)} .
$$

Therefore, (8.2) can not be satisfied simultaneously for $i=1,2$. The case $\alpha_{2}>\alpha_{1}$, $\beta_{2}>\beta_{1}$ is handled in a similar fashion.

Strictly positive solutions exist if the assumptions of the following corollary hold:

Corollary 8.3 Let $E_{i} e^{\lambda_{i} t}, i=1,2$ be the exponential solutions defined in Section 7. If both $E_{i} e^{\lambda_{i} t}, i=1,2$ are unstable, then system (6.1) (with $n=2$ ) has at least one strictly positive exponential solution.

Proof. Applying Theorem 7.2 we have

$$
\begin{aligned}
R_{2}^{1}= & \left(\lambda_{1}+2 \mu+\sigma\right)\left(\alpha_{2} \beta_{1}+\beta_{1} \alpha_{2}-2 \alpha_{1} \beta_{1}\right) \\
& -\frac{\alpha_{1} \beta_{1}}{\alpha_{1}+\beta_{1}}\left(\alpha_{2} \beta_{1}+\beta_{2} \alpha_{1}-2 \alpha_{2} \beta_{2}\right) \\
> & 0 \\
R_{1}^{2}= & \left(\lambda_{2}+2 \mu+\sigma\right)\left(\alpha_{2} \beta_{1}+\beta_{1} \alpha_{2}-2 \alpha_{2} \beta_{2}\right) \\
& -\frac{\alpha_{1} \beta_{1}}{\alpha_{2}+\beta_{2}}\left(\alpha_{2} \beta_{1}+\beta_{2} \alpha_{1}-2 \alpha_{1} \beta_{1}\right)
\end{aligned}
$$

$>0$. 
¿From (8.3) we have that

$$
\alpha_{2} \beta_{1}+\beta_{1} \alpha_{2}-2 \alpha_{i} \beta_{i}>0 . \quad i=1,2
$$

Hence, $\alpha_{2} \beta_{1} \neq \beta_{1} \alpha_{2}$ and also

$$
\frac{\beta_{1}}{\alpha_{1}+\beta_{1}} \neq \frac{\beta_{2}}{\alpha_{2}+\beta_{2}}
$$

We suppose without loss of generality that

$$
\frac{\beta_{1}}{\alpha_{1}+\beta_{1}}<\frac{\beta_{2}}{\alpha_{2}+\beta_{2}}
$$

and let the functions $\lambda_{i}(T)>\mu, T \in\left[T_{1}, T_{2}\right], i=1,2$, be defined by the implicit relationship

$$
\lambda_{i}(T)+\mu-k\left(\frac{\alpha_{i} T}{\lambda_{i}(T)+2 \mu+\sigma+\alpha_{i} T}+\frac{\beta_{i}(1-T)}{\lambda_{i}(T)+2 \mu+\sigma+\beta_{i}(1-T)}\right)=0
$$

where $T_{1}=\frac{\beta_{1}}{\alpha_{1}+\beta_{1}}$ and $T_{2}=\frac{\beta_{2}}{\alpha_{2}+\beta_{2}}$. Then from implicit function theory we see that $\lambda_{i}(T), i=1,2$ are well defined and are continuous functions of $T$ on $\left[T_{1}, T_{2}\right]$. Furthermore, since

$$
\begin{aligned}
\lambda_{1}^{1} & +\mu-k\left(\frac{\alpha_{1} T_{1}}{\lambda_{1}^{1}+2 \mu+\sigma+\alpha_{1} T_{1}}+\frac{\beta_{1}\left(1-T_{1}\right)}{\lambda_{1}^{1}+2 \mu+\sigma+\beta_{1}\left(1-T_{1}\right)}\right) \\
& =z_{1}^{1}-k\left(\frac{a_{11}}{z_{1}^{1}+h+a_{11}}+\frac{b_{11}}{z_{1}^{1}+h+b_{11}}\right) \\
& =z_{1}^{1}-\frac{2 k a_{11}}{z_{1}^{1}+h+a_{11}} \\
& =0
\end{aligned}
$$

we have that

$$
\lambda_{1}^{1}=\lambda_{1}\left(T_{1}\right)
$$

¿From (7.10) and (8.3) we deduce that

$$
\lambda_{2}\left(T_{1}\right)=\lambda_{2}^{1}>\lambda_{1}^{1}=\lambda_{1}\left(T_{1}\right)
$$


and an application of the same argument to $T_{2}$ leads to

$$
\lambda_{1}\left(T_{2}\right)>\lambda_{2}\left(T_{2}\right) .
$$

¿From the continuity of $\lambda_{i}(T),(8.4)$, and (8.5) there is a $T^{*} \in\left(T_{1}, T_{2}\right)$ such that

$$
\lambda_{1}\left(T^{*}\right)=\lambda_{2}\left(T^{*}\right)
$$

The existence of strictly positive exponential solutions follows from Lemma 8.1.

Remark 1 If both $E_{i} e^{\lambda_{i} t}, i=1,2$ are stable, then using similar arguments as those in the proof of Corollary (8.3), we conclude that there is a strictly positive exponential solution. However, it is not clear if we can select parameters from the $\alpha_{i}$ 's and the $\beta_{i}$ 's so that $E_{i} e^{\lambda_{i} t}, i=1,2$ are both stable. We believe that this is not possible on biological grounds.

Remark 2 If one of the solutions $E_{i} e^{\lambda_{i} t}$ is stable and the other unstable then either $\lambda_{1}\left(T_{i}\right)>\lambda_{2}\left(T_{i}\right)$ or $\lambda_{2}\left(T_{i}\right)>\lambda_{1}\left(T_{i}\right), i=1,2$. However, we have not shown that the curves $\lambda_{1}(T)$ and $\lambda_{2}(T)$ do not intersect, that is, that there is no strictly positive exponential solution. Numerical studies of this equation have not revealed possible crossings (see Figure 1).

Remark 3 Finally, it is not clear if the strictly positive exponential solution is unique. However, our numerical analysis has only shown one intersection (see Figure 2).

\section{Conclusions}

In this article, we have formulated and partially analyzed pair-formation models for multiple groups with general pairing rates, arbitrary mixing probabilities, and constant recruitment rates. Despite the complexity of the system, the dynamics 
CASTILLO-CHAVEZ, C. HUANG, W. LI, J.

are relatively simple because of its monotonicity properties. If we assume that the average partnership durations are identical for all groups, we also compute the stable pairing distributions. We have also analyzed models with nonconstant recruitment ( that is, constant per-capita recruitment rates) that give rise to homogeneous systems of degree one. In this case the situation becomes more complicated, yet we are able to classify the stability of all nonstrictly positive boundary exponential solutions,. However, it is unclear to us what are necessary and sufficient conditions for the existence and stability of strictly interior exponential solutions. We cannot rule out oscillations. We feel that these mathematical problems are of enough relevance in demography, epidemiology, and population dynamics that they deserve further investigation and, consequently, we hope that mathematicians will become interested in them.

\section{Acknowledgments}

This research was partially supported by NSF grant DEB-925370 to Carlos Castillo-Chavez and by the U.S. Army Research Office through the Mathematical Science Institute of Cornell University (contract DAAL03-91-C-0027). This article was completed while Carlos Castillo-Chavez was a Fellow at the Department of Ecology and Evolutionary Biology in Princeton University. 


\section{References}

[1] American Heritage Dictionary of The English Language (1992). Third Ed. Houghton Miflin Co., Boston and New York.

[2] Anderson, R.M., S.P. Blythe, S. Gupta and E. Konings (1989), The transmission of the Human Immunodeficiency Virus Type I in the male homosexual community in the United Kingdom: the influence of changes in sexual behavior, Phil. Trans., Roy. Soc. London, B 325, 145-198.

[3] Bailey, N.T.J. (1975). The Mathematical Theory of Infectious diseases and its applications. Griifin: London.

[4] Blythe, S.P., and C. Castillo-Chavez (1989). Like-with-like preference and sexual mixing models. Math. Biosci. 96:221-238.

[5] Blythe, S.P., C. Castillo-Chavez, J. Palmer \& M. Cheng (1991), Towards a unified theory of mixing and pair formation, Math. Biosci., 107:379-405.

[6] Blythe, S.P., C. Castillo-Chavez, and S. Busenberg. (1994). Affinity and paired-event probability. Biometrics Unit Technical Report BU-1084-M, Cornell University. Submitted, Math. Biosci..

[7] Busenberg, S., and C. Castillo-Chavez (1989). Interaction, pair formation and force of infection terms in sexually-transmitted diseases. In: C. Castillo-Chavez (ed.), Mathematical and statistical approaches to AIDS epidemiology, pp.289300. Lecture Notes in Biomathematics, Vol. 83, Springer-Verlag: Berlin.

[8] Busenberg, S., and C. Castillo-Chavez (1991), A general solution of the problem on mixing subpopulations, and its application to risk- and age-structured epidemic models for the spread of AIDS. IMA J. of Mathematics Applied in Med. and Biol., 8, 1-29.

[9] Castillo-Chavez, C. (ed.) (1989). Mathematical and statistical approaches to AIDS epidemiology. Lecture Notes in Biomathematics, Vol. 83, Springer- 
Verlag: Berlin.

[10] Castillo-Chavez, C., and S. P. Blythe (1989). Mixing framework for social/ sexual behavior. In C. Castillo-Chavez (ed.), Mathematical and Statistical Approaches to AIDS Epidemiology, 275-288. Lecture Notes in Biomathematics, Vol. 83, Springer-Verlag: Berlin.

[11] Castillo-Chavez, C. \& Busenberg, S. (1991), On the solution of two-sex problem. In S. Busenberg and M. Martelli (eds.) Proceedings of the International Conference on Differential Equations and Applications to Biology and Population Dynamics, Lecture Notes in Biomathematics, 80-98, Springer-Verlag, Berlin-Heidelberg-New York-London-Paris-Tokyo-Hong Kong, Barcelona, Budapest.

[12] Castillo-Chavez, C., S. Busenberg \& K. Gerow (1991), Pair formation in structured populations, In: J. Goldstein, F. Kappel, and W. Schappacher (eds.), Differential Equations with Applications in Biology, Physics and Engineering, 47-65, Marced Dekker: New York.

[13] Castillo-Chavez, C. and W. Huang (1994), The logistic equation revisited: the two-sex case (manuscript).

[14] Castillo-Chavez, C., S-F. Shyu, G. Rubin, and D. Umbauch (1992) On the estimation problem of mixing/pair formation matrices with applications to models for sexually-transmitted diseases. In: N.P. Jewell, K. Dietz, and V.T. Farewell (eds.), AIDS Epidemiology: Methodology Issues, pp.384-402. Birkhäuser: Boston.

[15] Castillo-Chavez, C. and H.R. Thieme (1993). Asymptotically autonomous epidemic models. In O. Arino, M. Kimmel (eds.) Proc. Third Intern. Conf. on Mathematical Population Dynamics (to appear)

[16] Castillo-Chavez, C., Velasco-Hernàndez, J. X.\& Fridman, S. (1994). Modeling Contact Structures in Biology. In S. A. Levin (ed.), Frontiers of Theoretical 
Biology. Lecture Notes in Biomathematics 100 (in press). Berlin, Heidelberg, New York, London, Paris, Tokyo, Hong Kong, Barcelona, Budapest: SpringerVerlag.

[17] Dietz, K. (1988). On the transmission dynamics of HIV. Math. Biosci., 90:397414.

[18] Dietz, K \& Hadeler, K.R.(1988), Epidemiological models for sexually transmitted diseases, J. Math. Biol. 26, 1-25.

[19] Euler, L. (760), Résérches generales sur la mortalité et la multiplication du genre humaine. Histoire de l'Académie Royale de Sciences et Belles Lettres $16: 144-164$.

[20] Feller, W. (1941, On the integral equation of rebewal theory. Annals of Mathematical Statistics 12:243-267.

[21] Fredrickson, A.G.(1971), A mathematical theory of age structure in sexual populations: Random mating and monogamous marriage models, Math. Biosci. $20,117-143$.

[22] Gupta, S., R.M. Anderson and R.M. May (1989), Networks of sexual contacts: Implications for pattern of spread of HIV., J. AIDS. 3, 1-11.

[23] Hadeler, K.P. (1989a). Pair formation in age-structured populations. Acta Applicandae Mathematicae, 14:91-102.

[24] Hadeler, K.P. (1989b). Modeling AIDS in structured populations. The 47th Session of the International Statistical Institute, Paris, August/September. Conf. Proc., C1-2.1:83-99.

[25] Hadeler, K.P (1990), Homogeneous delay equations and models for pair formation, CDSNS90-17, Center for Dynamical Systems and Nonlinear Studies, Georgia Institute of Technology.

[26] Hadeler, K.P., and K. Nagoma (1990). Homogeneous models for sexuallytransmitted diseases. Rocky Mountain Journal of Mathematics, 20:967-986. 


$$
\text { CASTILLO-CHAVEZ, C. HUANG, W. LI, J. }
$$

[27] Hadeler, K.P., Waldstätter, R., and Wörz-Busekros A. (1988). Models for pair-formation in bisexual populations. Journal of Math. Biol., 26:635-649.

[28] Hadeler, K.P (1992), Periodic solutions of homogeneous equations, J. Diff. Equations, 95:183-202.

[29] Hethcote, H.W., and J.A. Yorke (1984). Gonorrhea Transmission Dynamics and Control. Lecture Notes in Biomathematics, Vol. 56. Springer-Verlag: New York.

[30] Hirsch, M. W., Systems of differential equations which are competitive or cooperative. I: Limit sets, SIAM J. Math. Anal., 13 (2), 1982, 167-179.

[31] Hoppensteadt, F. (1975). Mathematical Theories of Populations: Demographics, Genetics and Epidemics. SIAM Regional Conference Series in Applied Math., 20, Philadelphia.

[32] Hsu Schmitz, S-F, Some theories, estimation methods and applications of marriage functions in demography and epidemiology, $\mathrm{Ph} \mathrm{D}$ Cornell University dissertation, 1993.

[33] Hsu Schmitz, S-F. \& C. Castillo-Chavez (1992), Completion of mixing matrices for nonclosed social networks, In: Proceedings to the First World Congress of Nonlinear Analysis, Tampa, Florida, August 19-26, 1992 (in press).

[34] Hsu Schmitz, S.F, Busenberg, S. \& Castillo-Chavez, C. (1993), On the evolution of marriage functions: It takes two to tango, Biometrics Unit Technical Report BU-1210-M, Cornell University, Ithaca, New York.

[35] Hsu Schmitz, S-F., and C. Castillo-Chavez (1994). Parameter estimation in non-closed social networks related to the dynamics of sexually-transmitted diseases. In: E. H. Kaplan and M. L. Brandeau (eds.), Modeling the AIDS Epidemic: Planning, Policy, and Prediction, 533-559. Raven Press, New York

[36] Huang, W., K.Cooke and C. Castillo-Chavez (1992), Stability and bifurcation for a multiple group model for the dynamics of HIV/AIDS transmission, SIAM 
J. Appl. Math., 52(3), 835-854.

[37] Hyman, J.M. and E.A. Stanley (1989), The effect of social mixing patterns on the spread of AIDS, Lect. In: C. Castillo-Chavez, S. A. Levin, and C. Shoemaker (eds.), Mathematical Approaches to Problems in Resource Management and Epidemiology, 190-219. Lecture Notes in Biomathematics 81, SpringerVerlag, Berlin, Heidelberg, New York.

[38] Inaba, H. (1988), A semigroup approach to the strong ergodic theorem of the multistate stable population process, Math. Pop. Stud., 1(1):49-77.

[39] Jacquez, J.A., C.P. Simon, J. Koopman, I. Sattenspei and T. Perry (1988), Modelling and analyzing HIV transmission: the effect of contact patterns, Math. Biosci., 92, 119-199.

[40] Jacquez, J.A., C.P. Simon and J. Koopman (1989), Structured mixing: heterogeneous mixing by the definition of mixing groups. In C. Castillo-Chavez (ed.), Mathematical and Statistical Approaches to AIDS Epidemiology, 301-315. Lecture Notes in Biomathematics, Vol. 83, Springer-Verlag.

[41] Jewell, N.P., K. Dietz, and V.T. Farewell (1991). AIDS epidemiology: Methodological issues. Birkhäuser: Boston.

[42] Kendall, D.G. (1949), Stochastic processes and population growth, Royal Statistical Society, Ser. B2:230-264.

[43] Keyfitz, N. (1949), Introduction to the Mathematics of Populations with revisions, Addison-Wesley, Reading, MA. and Menlo Park, CA.

[44] Keyfitz, N. (1977), The mathematics of sex and marriage, Proceedings of the Sixth Berkeley Symposium on Mathematical Statistics and Probability, Vol. IV: Biology and Health, 89-108.

[45] Lotka, A. J. (1907), Relation between birth rates and death rates, Science $N$. S., 26:21-22. 
CASTILLO-CHAVEZ, C. HUANG, W. LI, J.

[46] Lotka, A. J. (1922), The stability of the normal age distribution, Proceedings of the National Academy of the Sciences, 8:339-345.

[47] Lubkin, S \& Castillo-Chavez, C., A pair formation approach to modeling inheritance of social traits, Biometrics Unit Technical Report BU-1182-M, Cornell University, Ithaca, New York.

[48] Lubkin, S., S-F. Hsu Schmitz \& Castillo-Chavez, C. (1992), A framework for modeling inheritance of social traits, In: Proceedings to the First World Congress of Nonlinear Analysis, Tampa, Florida, August 19-26, 1992 (in press).

[49] Luo, X., and C. Castillo-Chavez (1993). Limit behavior of pair-formation for a large dissolution rate. Journal of Mathematical Systems, Estimation, and Control, 3(2):247-264.

[50] Malthus, TR.(1978), An Essay on the Principle of Population (1st Ed), J Johnson in St Paul's Churchyard, London.

[51] May, R.M. and R.M. Anderson. (1989). The transmission dynamics of human immunodeficiency virus (HIV). Phil. Trans. R. Soc. London, B, 321, 565-607.

[52] McFarland, D. (1972), Comparison of alternative marriage models. In: T. N. E. Greville (ed.), Population Dynamics, Academic Press, New York London: 89-106.

Math.Biosci., 52:227-240.

[53] Parlett, B. (1972), Can there be a marriage function? In: T. N. E. Greville (ed.), Population Dynamics, Academic Press, New York London: 107-135.

[54] Pollard, J.H. (1973), Mathematical models for the growth of human populations, Chapter 7: The two sex problem, Cambridge University Press.

[55] Ross, R. (1911), The prevention of malaria (2nd ed.), John Murray, London.

[56] Sattenspiel, L (1987a). Population structure and the spread of disease. American Journal of Physical Anthropology, 73, 251-265. 
[57] Sattenspiel, L (1987b). Epidemics in nonrandomly mixing populations. Huamn Biology, 59, 411-438.

[58] Sattenspiel, L. and C. Castillo-Chavez. (1990). Environmental context, social interactions, and the spread of HIV. American Journal of Human Biology, 2, 397-417.

[59] Sattenspiel L. and C. Simon (1988). The spread and Persitence of Infectious Diseases in Structured Populations, Math. Bios., 90, 341-366.

[60] Sharpe, F. R. and Lotka, A. J. (1911),A problem in age distribution, Philosophical Magazine, Ser. 6 21:435-438.

[61] Smith, D. and N. Keyfitz (1977), Mathematical Demography, Biomathematics 6, Springer-Veralg, Berlin, Heidelberg, New York.

[62] Smith Hal L., and H.R. Thieme (1990), Quasi-convergence and stability for strongly order preserving semiflows, SIAM J. Math. Anal, Vol 21, No.3, 673692.

[63] Thieme, H.R. (1992): Convergence results and a Poincaré-Bendixson trichotomy for asymptotically autonomous differential equations. J. Math. Biol. 30, $755-763$

[64] Thieme, H.R. (1993a): Asymptotically autonomous differential equations in the plane, Rocky Mt. J. Math. (to appear)

[65] Thieme, H.R, (1993b), Asymptotically autonomous differential equations in the plane II. Stricter Poincaré-Bendixson type results, Diff. Integral Eq. (to appear)

[66] Waldstätter, R. (1989). Pair formation in sexually transmitted diseases. In: C. Castillo-Chavez (ed.), Mathematical and Statistical Approaches to AIDS Epidemiology, pp.260-274. Lecture Notes in Biomathematics, Vol. 83, SpringerVerlag: Berlin. 


\section{APPENDIX}

\section{Proof of Lemma 8.1}

Let $x_{i}+p_{i}=u_{i}, y_{i}+q_{i}=v_{i}, i=1,2$ and let

$$
T=\frac{\sum_{k}^{2} \beta_{k} y_{k}}{\sum_{k}^{2}\left(\alpha_{k} x_{k}+\beta_{k} y_{k}\right)},
$$

then, as in the proof the proof of Theorem 7.2, we obtain the following system (which is equivalent to (8.1)):

$$
\begin{gathered}
(\lambda+\mu) u_{i}=k\left(p_{i}+q_{i}\right), \quad i=1,2 \\
(\lambda+\mu) v_{i}=k\left(p_{i}+q_{i}\right), \quad i=1,2 \\
\alpha_{i} T u_{i}=\left(\lambda+2 \mu+\sigma+\alpha_{i} T\right) p_{i}, \quad i=1,2 \\
\beta_{i}(1-T) v_{i}=\left(\lambda+2 \mu+\sigma+\beta_{i}(1-T) q_{i}, \quad i=1,2 .\right.
\end{gathered}
$$

From (A.1) and (A2) we have that

$$
\lambda+\mu>0 \quad \text { and } \quad u_{i}=v_{i} .
$$

(A.3) and (A.4) yield

$$
p_{i}=\frac{\alpha_{i} T u_{i}}{\lambda+2 \mu+\sigma+\alpha_{i} T}, \quad q_{i}=\frac{\beta_{i}(1-T) u_{i}}{\lambda+2 \mu+\sigma+\beta_{i}(1-T)},
$$

while (A.1) and (A.6) imply that

$$
\lambda+\mu-k\left(\frac{\alpha_{i} T}{\lambda+2 \mu+\sigma+\alpha_{i} T}+\frac{\beta_{i}(1-T)}{\lambda+2 \mu+\sigma+\beta_{i}(1-T)}\right)=0 .
$$

Furthermore, (A.6) gives that

$$
x_{i}=u_{i}-p_{i}=z \frac{u_{i}}{z+\alpha_{i} T}, \quad y_{i}=u_{i}-q_{i}=z \frac{u_{i}}{z+\beta_{i}(1-T)},
$$


where $z=\lambda+2 \mu+\sigma$. It follows that

$$
\begin{gathered}
\sum_{k}^{2} \alpha_{i} x_{i}=z \sum_{k}^{2} \frac{\alpha_{k} u_{k}}{z+\alpha_{i} T}, \\
\sum_{k}^{2} \beta_{i} x_{i}=z \sum_{k}^{2} \frac{\beta_{k} u_{k}}{z+\beta_{i}(1-T)} .
\end{gathered}
$$

Hence, we need to choose $u_{i}>0, i=1,2$ such that

$$
\begin{gathered}
z \sum_{k}^{2} \frac{\alpha_{k} u_{k}}{z+\alpha_{i} T}=T \\
z \sum_{k}^{2}\left(\frac{\alpha_{k}}{z+\alpha_{i} T}+\frac{\beta_{k}}{z+\beta_{i}(1-T)}\right) u_{k}=1 .
\end{gathered}
$$

Equations (A.7) and (A.8) give two straight lines in the $u_{1}-u_{2}$ plane which intersect the $u_{2}$ axis at the points

$$
\left(0, \frac{T\left(z+\beta_{2}(1-T)\right.}{\beta_{2} z}\right) \quad \text { and } \quad\left(0, \frac{\left(z+\alpha_{2} T\right)\left(z+\beta_{2}(1-T)\right.}{\left(\alpha_{2}+\beta_{2}\right) z+\alpha_{2} \beta_{2}}\right),
$$

respectively, and they intersect with the $u_{1}$ axis at the points

$$
\left(\frac{T\left(z+\beta_{1}(1-T)\right.}{\beta_{1} z}, 0\right) \quad \text { and } \quad\left(\frac{\left(z+\alpha_{1} T\right)\left(z+\beta_{1}(1-T)\right.}{\left(\alpha_{1}+\beta_{1}\right) z+\alpha_{1} \beta_{1}}, 0\right),
$$

respectively. Therefore, the System (A.7)-(A.8) has a solution with $u_{1}>0, u_{2}>0$ if and only if

$$
\begin{aligned}
& \frac{T}{\beta_{2}}>\frac{z+\alpha_{2} T}{\left(\alpha_{2}+\beta_{2}\right) z+\alpha_{2} \beta_{2}} \\
& \frac{T}{\beta_{1}}>\frac{z+\alpha_{1} T}{\left(\alpha_{1}+\beta_{1}\right) z+\alpha_{1} \beta_{1}},
\end{aligned}
$$

or

$$
\begin{aligned}
& \frac{T}{\beta_{2}}<\frac{z+\alpha_{2} T}{\left(\alpha_{2}+\beta_{2}\right) z+\alpha_{2} \beta_{2}} \\
& \frac{T}{\beta_{1}}>\frac{z+\alpha_{1} T}{\left(\alpha_{1}+\beta_{1}\right) z+\alpha_{1} \beta_{1}} .
\end{aligned}
$$

The case where $\alpha_{1}=\alpha_{2}, \beta_{1}=\beta_{2}$ is trivial. 
When (A.9) holds we have

$$
\begin{aligned}
& T\left(\alpha_{2}+\beta_{2}\right) z+T \alpha_{2} \beta_{2}>\beta_{2} z+\alpha_{2} \beta_{2} T \\
& T\left(\alpha_{1}+\beta_{1}\right) z+T \alpha_{1} \beta_{1}>\beta_{1} z+\alpha_{1} \beta_{1} T
\end{aligned}
$$

that is equivalent to

$$
\frac{\beta_{2}}{\alpha_{2}+\beta_{2}}<T<\frac{\beta_{1}}{\alpha_{1}+\beta_{1}}
$$

When (A.10) holds we have

$$
\begin{aligned}
& T\left(\alpha_{2}+\beta_{2}\right) z+T \alpha_{2} \beta_{2}<\beta_{2} z+\alpha_{2} \beta_{2} T \\
& T\left(\alpha_{1}+\beta_{1}\right) z+T \alpha_{1} \beta_{1}>\beta_{1} z+\alpha_{1} \beta_{1} T
\end{aligned}
$$

that is equivalent to

$$
\frac{\beta_{2}}{\alpha_{2}+\beta_{2}}>T>\frac{\beta_{1}}{\alpha_{1}+\beta_{1}}
$$

Hence, the proof of Lemma (8.1) is complete. 